\title{
Estado actual del diagnóstico y presentaciones clínicas de enfermedad celíaca. Estudio multicéntrico
}

\author{
Paulina Canales $\mathbf{R}^{1}$, Magdalena Araya $\mathbf{Q}^{2}$, \\ Francisco Alliende $\mathbf{G}^{3}$, Bessie H unter $\mathbf{M}^{4}$, \\ Teresa Alarcón $\mathbf{0}^{5}$, Eduardo Chávez $\mathrm{S}^{6}$. \\ Diagnosis and clinical presentations \\ of celiac disease. A multicenter study
}

Background: Ample use of serological markers of high sensitivity and specificity led to relevant changes in the epidemiology of celiac disease. The impact of these changes in our country is poorly known. Aim: To assess the diagnostic procedures, clinical presentations and follow up of celiac disease as conducted in current pediatric practice. Material and methods: A multicentric retrospective study of patients diagnosed between 2000 and 2005 in five pediatric hospitals in Santiago, Chile. Data was obtained from clinical records, recorded in electronic spreadsheets and analyzed by descriptive statistics. Results: Seventy four of 83 identified patients fulfilled the inclusion criteria and were analyzed. Mean time to reach the diagnosis was 2.1 years. Cases younger than 10 years presented digestive manifestations such as chronic diarrhea and abdominal distension. Twenty one percent of older patients had atypical presentations (mainly short stature, refractory anaemia). Ten percent of cases were screened because a first degree relative had celiac disease. All patients had significant duodenal/jejunal lesion. IgA-antiendomysial antibodies $(\mathrm{n}=65)$ and IgA-antigliadin antibodies $(\mathrm{n}=23)$ were the most commonly used screening tests used but often, they were not available for follow up. A second biopsy was planned in all patients but only 26 had it due to repeated dietary transgressions, often due to unnoticed consumption of gluten in poorly labeled products. Conclusions: Digestive manifestations were the main presentation form for celiac disease among patients under 10 years of age. Atypical symptoms become relevant in patients older than 10 years. Antiendomysial and antitransglutaminase antibody measurement should be incorporated for routine screening and follow up of celiac disease in public hospitals. To improve food labeling about their gluten content is needed (Rev Méd Chile 2008; 136: 296-303).

(Key words: Celiac disease)

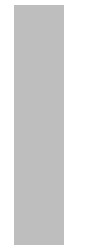

\footnotetext{
Recibido el 4 de junio, 2007. Aceptado el 19 de noviembre, 2007.

Financiamiento: Este trabajo fue financiado parcialmente por CINUT.

${ }^{1}$ Servicio de Pediatría, Hospital Exequiel González Cortés. ${ }^{2}$ Instituto de Nutrición y Tecnología de los Alimentos (INTA), Universidad de Chile. ${ }^{3}$ Servicio de Gastroenterología, Hospital Roberto del Río. ${ }^{4}$ Servicio de Gastroenterología, Hospital Luis Calvo Mackenna. ${ }^{5}$ Servicio de Gastroenterología, Hospital San Juan de Dios. ${ }^{6}$ Servicio de Gastroenterología, Hospital San Borja Arriarán.
}

Correspondencia a: Magdalena Araya. INTA, Universidad de Chile. Macul 5540, Santiago. Fono: 9781468. Fax: 2214030. E mail: maraya@inta.cl 
$\mathrm{L}^{\mathrm{a}}$ a enfermedad celíaca (EC) es una enteropatía autoinmune de base genética, caracterizada por presentar grados variables de daño en la mucosa intestinal ${ }^{1-2}$. Esta condición aparece en individuos genéticamente susceptibles, que han sido expuestos al gluten, una proteína contenida en trigo, cebada y centeno ${ }^{2}$. Hace una década atrás era considerada una condición de prevalencia más bien baja, que afectaba el tracto digestivo, principalmente en niños; hoy se sabe que es una condición de alta frecuencia y que puede afectar tanto a niños como adultos ${ }^{3}$.

Este cambio de escenario se produjo como resultado del uso masivo de marcadores serológicos de alta sensibilidad y especificidad, los anticuerpos antiendomisio (EMA) y anticuerpos antitransglutaminasa (TTG) ${ }^{1-4}$. Su utilización como método de rastreo (screening) reveló que existen pacientes celíacos con grados variables de enteropatía, que los síntomas digestivos pueden ser poco prominentes o bien que, a nivel clínico, puede haber franco predominio de manifestaciones extraintestinales. Previo al uso de estos marcadores, este tipo de pacientes no eran diagnosticados; un estudio realizado en 1993 mostró que 75\% de los casos diagnosticados presentaban diarrea, hoy en cambio, 50\% de los casos se diagnostica a través de estudios de rastreo realizados en grupos considerados de riesgo, o sea, individuos activamente buscados que no consultan por sintomatología bien establecida $^{5}$. Cuando estos grupos con presentaciones atípicas se incorporaron al análisis de prevalencia, ésta aumentó a 1:100 - 1:5006-10, contrastando con cifras previas de 1:1.800 - 1:3.000, basadas en el estudio de casos que consultaban con presentaciones digestivas y eran diagnosticados solamente por biopsia intestinal.

En la actualidad, la enfermedad se está reclasificando, habiendo diferencias en los términos según los autores. Según los criterios emanados de un grupo de expertos reunidos por el National Institute of Health (NIH), se clasifica en 4 grupos de acuerdo a la presentación clínica y magnitud del daño de la mucosa; la forma típica (enteropatía grave con predominio de síntomas digestivos), atípica (enteropatía con predominio de síntomas extradigestivos, por ejemplo anemia ferropriva, osteoporosis, condiciones autoinmunes, etc.), silente (enteropatía con síntomas mínimos o asinto- máticos, frecuentemente diagnosticados por estudio serológico) y potenciales (enteropatía mínima sin síntomas clínicos con antecedentes histológicos o serológicos positivos en algún momento de la historia clínica) ${ }^{11-13}$; aunque esta última es la categoría más controvertida, es mantenida por autores considerados líderes en el tema11-13. La mayoría de los estudios de rastreo incluyen población adulta $9,14,15$ y no se sabe hasta qué punto el uso de herramientas serológicas actualmente ha cambiado el escenario clínico en la población pediátrica, qué tan frecuente son las formas atípicas y cómo se está realizando el diagnóstico en la población pediátrica. Con estos antecedentes se definió el objetivo de este estudio: evaluar el manejo de la enfermedad celíaca en los servicios pediátricos de 5 hospitales públicos de Santiago, caracterizando las presentaciones clínicas (proporción de formas típicas y atípicas), los marcadores serológicos más frecuentemente utilizados para guiar el diagnóstico y seguimiento, el número de biopsias realizadas para el diagnóstico y el tratamiento y seguimiento de la enfermedad en los centros participantes.

\section{Material Y MÉTODO}

Este estudio fue multicéntrico, de carácter retrospectivo e incluyó a los pacientes menores de 15 años que fueron diagnosticados durante los años 2000-2005, en quienes se realizó algún tipo de rastreo serológico y biopsia intestinal, en los hospitales Exequiel González Cortés, Roberto del Río, San Juan de Dios, San Borja Arriarán y Luis Calvo Mackenna. La información recolectada corresponde a datos secundarios en los centros participantes, por lo que se mantuvo el anonimato de los participantes, pero no se solicitó consentimiento informado; el protocolo fue aprobado por el Comité de Ética del Instituto de Nutrición y Tecnología de los Alimentos (INTA), Universidad de Chile.

El diagnóstico de EC se basó en criterios actualmente establecidos por la European Society for Pediatric Gastroenterology, Hepatology and Nutrition (ESPGHAN) y en la North American Society for Gastroenterology, Hepatology and Nutrition (NASGHAN) ${ }^{16-18}$, es decir, al menos una biopsia intestinal (biopsia I, diagnóstica) y al 
menos un test serológico positivo, anticuerpo antigliadina ((AGA) IgA o IgG, IgA-EMA o IgATTG). La detección de anticuerpos fue realizada en cada centro participante siguiendo sus técnicas de rutina. Esto introdujo variabilidad en las técnicas, kits utilizados y formas de expresión de resultados, lo que no permitió analizar comparativamente los resultados según puntos de corte o unidades obtenidas por los pacientes en estos exámenes. Los datos clínicos recolectados incluyeron edad, sexo, edad al diagnóstico, tiempo transcurrido entre el inicio de los síntomas y el diagnóstico y manifestaciones clínicas (diarrea, distensión abdominal, dolor abdominal, vómitos, anorexia, estado nutricional, constipación, crisis celíaca, intestino irritable, anemia ferropriva resistente a tratamiento, defectos del esmalte dental, aftas orales, retraso puberal), condiciones autoinmunes (hepatitis, tiroiditis, neuropatía, síndrome de Sjögren), dermatitis herpetiforme, trastornos de la personalidad, epilepsia, síndrome de Down, síndrome de Turner, síndrome de Williams, déficit de IgA, familiares en primer grado con EC. El registro de los datos antropométricos respetó la denominación de los registros originales, clasificando como falla de crecimiento (lactantes con falla principalmente de Peso/Edad), desnutrición crónica (lactantes con compromiso de Peso/Edad y Talla/Edad), talla baja (escolares y niños mayores con falla principalmente de Talla/Edad). Los datos fueron registrados en formularios ad-hoc, vaciados a una planilla Excel y analizados usando estadística descriptiva (STAT 6.0). Dada que también había una variabilidad considerable en los informes histológicos y la mayoría de la información registrada no permitía ser clasificada según los criterios de Marsh, este aspecto se analizó sólo según hubiera lesiones intensas, moderadas o inespecíficas.

El análisis de resultados se realizó mediante estadística simple, descriptiva, incluyendo porcentajes, promedios o medianas según los datos tuvieran distribución normal o no y chi cuadrado.

\section{Resultados}

Durante el período de 5 años analizado se realizó una evaluación diagnóstica en 83 pacientes, de los cuales 74 cumplieron con los criterios de inclusión y constituyeron la población sometida a análisis. Las causas de exclusión del análisis fueron: en siete casos $(9,5 \%)$ no se les realizó ningún test serológico y el diagnóstico se basó en una biopsia intestinal (estos pacientes pertenecían a centros que no contaban con facilidades para realizar la determinación de anticuerpos), además, en un niño de 6,4 años con diarrea crónica y dolor abdominal, la biopsia mostró arquitectura vellositaria normal, con aumento de linfocitos intraepiteliales, la medición de EMA fue negativa y no se demostró déficit de IgA; el diagnóstico está aún en estudio. Finalmente, una niña de 6,3 años fue estudiada por diarrea crónica y desnutrición, sus EMA y TTG fueron positivos y la biopsia intestinal mostró daño moderado de la mucosa, con abundantes eosinófilos (que no fueron cuantificados) y se planteó el diagnóstico diferencial con alergia; la paciente consultó una vez y luego abandonó los controles. La proporción de casos diagnosticados en cada hospital participante varió entre 5 y 27 casos. La relación mujer:hombre, entre los casos diagnosticados, fue 1,3:1. La edad promedio de diagnóstico fue 2,8 años (P 25-75: 1,3-7,5 años) y el tiempo transcurrido entre el inicio de los síntomas y el diagnóstico fue de 2,1 años (promedio del grupo total) (Tabla 1). La distribución por edad fue asimétrica, con un conjunto por debajo de los 10 años y otro por encima de esta edad (mediana de 1,6 y 12,6 años en niños bajo y sobre 10 años, respectivamente).

Como era de esperar, la presentación típica (digestiva) fue característica de los niños menores (Figura 1), con predominio de diarrea crónica, retraso pondoestatural (signos de desnutrición aguda y retraso principalmente de Peso/Edad) y distensión abdominal como principales manifestaciones (Tabla 2). Las formas atípicas o extraintestinales se presentaron sólo en el grupo sobre los 10 años (típicas/atípicas vs bajo/sobre 10 años, $\chi^{2}=$ $12,15, \mathrm{p}<0,0005)$. En el grupo de niños mayores de 10 años, la anemia resistente al tratamiento con hierro y la talla baja (definida como compromiso de Talla/Edad) fueron las manifestaciones extraintestinales predominantes; un caso presentaba retraso puberal y síndrome de Turner, otro diarrea crónica y síndrome de Down, y un varón de 17 años consultó inicialmente por dispepsia tipo ulcerosa y anemia; debido a esta última se realizó un estudio de EMA, que fue positivo y la biopsia 
Tabla 1. C aracterísticas generales de 74 pacientes celíacos diagnosticados entre 2000-2005

\begin{tabular}{|lcc|}
\hline & $\begin{array}{c}<10 \text { años } \\
(\mathrm{n}=58)\end{array}$ & $\begin{array}{c}>10 \text { años } \\
(\mathrm{n}=16)\end{array}$ \\
\hline $\begin{array}{l}\text { Edad inicio síntomas } \\
\text { Promedio } \pm \text { DE (años) }\end{array}$ & $2,8 \pm 2,2$ & $13,3 \pm 2,6$ \\
$\quad$ Mediana (años) & 1,6 & 12,6 \\
Tiempo hasta el diagnóstico & $1,03 \pm 1,27$ & $4,3 \pm 4,2$ \\
$\quad$ Promedio \pm DE (años) & 6 & 46 \\
Mediana (meses) & $25 / 33$ & $7 / 9$ \\
Sexo & $49 / 6 / 3$ & $7 / 9 / 0$ \\
Masculino/femenino & & \\
Presentaciones típica/ atípica/silente & & \\
\hline
\end{tabular}

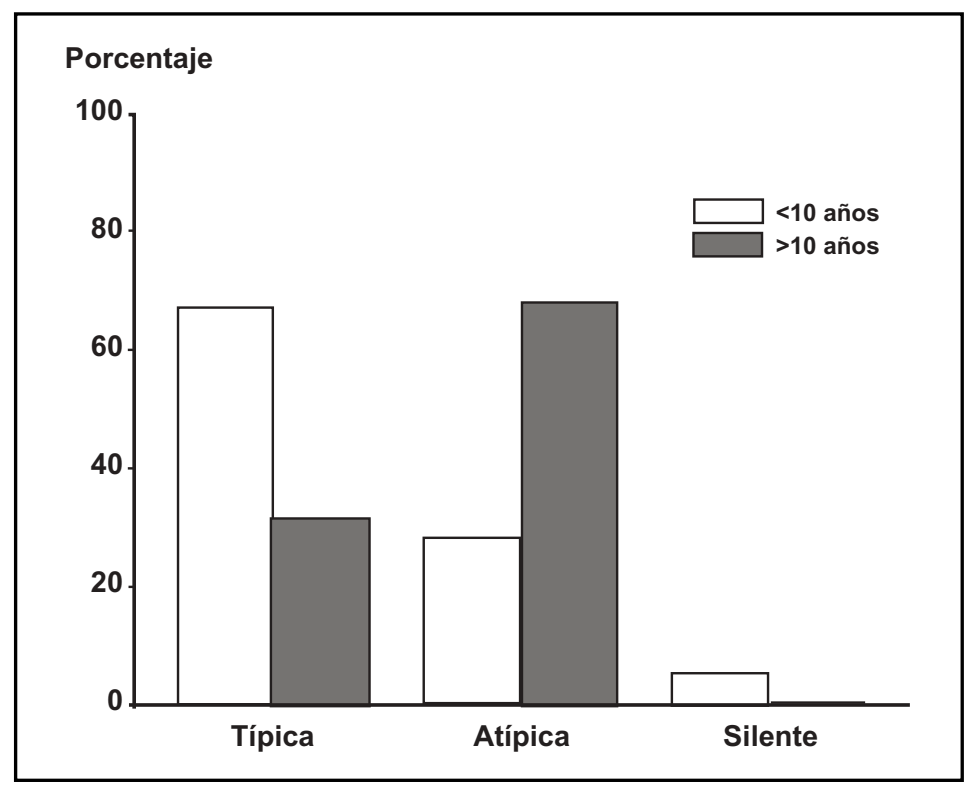

Figura 1. Presentaciones clínicas en 74 pacientes celíacos diagnosticados entre 2000-2005.

intestinal mostró daño grave de la mucosa. En $11 \%$ de los casos, la evaluación diagnóstica se realizó porque tenían un familiar de primer grado con enfermedad celíaca; en todos estos últimos, los anticuerpos fueron positivos y la biopsia intestinal confirmó el diagnóstico, pero tres se declaraban asintomáticos.
La alteración descrita en la mucosa intestinal fue intensa en todos los pacientes. Los exámenes serológicos más frecuentemente utilizados fueron IgA-EMA ( $\mathrm{n}=65)$; IgA-AGA ( $\mathrm{n}=23)$, IgA-TTG ( =23) e IgG-AGA $(n=4)$. En 8 pacientes la biopsia confirmó el diagnóstico, pero los EMA fueron negativos; cuatro de ellos presentaban deficiencia 
Tabla 2. M anifestaciones clínicas en 74 pacientes celíacos diagnosticados entre 2000-2005

\begin{tabular}{|c|c|c|c|c|c|c|}
\hline & $\begin{array}{c}\text { Total } \\
\mathrm{N}\end{array}$ & $\%$ & $\begin{array}{c}<10 \text { años } \\
n\end{array}$ & $\%$ & $\begin{array}{c}>10 \text { años } \\
\mathrm{n}\end{array}$ & $\%$ \\
\hline Diarrea crónica & 38 & 51 & 34 & 58 & 4 & 25 \\
\hline Falla de crecimiento ${ }^{1}$ & 29 & 39 & 26 & 44 & 3 & 19 \\
\hline Distensión abdominal & 14 & 19 & 12 & 20 & 2 & 12 \\
\hline Vómitos & 14 & 19 & 12 & 20 & 2 & 12 \\
\hline Anorexia & 10 & 13 & 9 & 15 & 1 & 6 \\
\hline Desnutrición crónica ${ }^{2}$ & 8 & 11 & 8 & 14 & - & - \\
\hline Crisis celíaca ${ }^{3}$ & 7 & 9 & 7 & 12 & - & - \\
\hline Dolor abdominal & 5 & 7 & 4 & 7 & 1 & 6 \\
\hline Constipación & 4 & 5 & 4 & 7 & & \\
\hline Estatura baja ${ }^{4}$ & 12 & 16 & 6 & 10 & 6 & 37 \\
\hline Anemia recurrente & 11 & 15 & 6 & 10 & 5 & 31 \\
\hline Enfermedad celíaca silente 5 & 3 & 4 & 3 & 5 & - & - \\
\hline Síndrome de Down & 1 & 1 & - & - & 1 & 6 \\
\hline Síndrome de Turner & 1 & 1 & 1 & 1 & - & - \\
\hline Otros $^{6}$ & 5 & 7 & 3 & 5 & 2 & 12 \\
\hline
\end{tabular}

${ }^{1}$ Falla de crecimiento se refiere a lactantes con compromiso de Peso/Edad. ${ }^{2}$ Desnutrición crónica se refiere a lactantes con compromiso Talla/Edad. ${ }^{3}$ Crisis celíaca entendida como descompensación grave de la enfermedad, con compromiso general intenso, diarrea profusa con desequilibrio hidroelectrolítico intenso, que puede acompañarse de tetania, edema, alteraciones hemorragíparas e incluso shock y compromiso sensorial. ${ }^{4}$ Estatura baja se refiere a preescolares y escolares con compromiso Talla/Edad. ${ }^{5}$ Enfermedad celíaca silente es la presentación clínica caracterizada por enteropatía con síntomas mínimos o asintomáticos, frecuentemente diagnosticados por screening serológico. ${ }^{6}$ Aftas orales, pica, retraso puberal, dispepsia ulcerosa, cefalea.

de IgA y los 4 restantes, todos menores de 2 años, tenían concentraciones séricas normales de IgA, lo que indica que la medición de EMA tuvo un error de $6,2 \%$ de los casos.

El criterio de seguimiento en todos los centros incluyó una segunda biopsia intestinal luego de 1 año de dieta libre de gluten; sin embargo, ésta se logró realizar en $26(34,7 \%)$ pacientes, que fueron aquellos que declararon (ellos o sus cuidadores) adherencia estricta a la dieta; en 9 de los 26 $(34,6 \%)$ las segundas biopsias revelaron histología normal, $12(46,2 \%)$ presentaban signos de daño leve y en $5(19,2 \%)$ los cambios continuaban siendo graves; en estos últimos, la encuesta alimentaria dirigida reveló frecuentes transgresiones a la dieta. Aunque en varios pacientes el seguimiento mostró que existían transgresiones voluntarias a la dieta, las historias clínicas revela- ron que, además, se producían transgresiones involuntarias por falta de conocimiento acerca del contenido de gluten en los alimentos elaborados de consumo habitual. En cuanto a los exámenes serológicos, no fue posible evaluar su rendimiento durante el seguimiento, debido a que en varios de los centros participantes se realizaban sólo para hacer el diagnóstico. Solamente en 7 pacientes $(9,5 \%)$ se efectuó una tercera biopsia a lo largo del seguimiento; en todos los casos ésta mostró reaparición del daño histológico grave.

\section{DisCUSIÓN}

Los resultados muestran que durante el período 2000-2005 se diagnosticaron 83 casos de EC en menores de 15 años atendidos en los 5 hospitales 
participantes. Estos hospitales reciben un alto número de consultas en Santiago; sin embargo, la cifra es claramente menor que la obtenida hace 15 años (1993), cuando se diagnosticaron 27 casos en un año en los mismos 5 hospitales (Daza W. Tesis de Magíster, INTA, Universidad de Chile). El hallazgo llama la atención, pero no necesariamente se debe interpretar como una disminución en la prevalencia de la enfermedad, ya que hoy día ha aumentado el número de familias que se controlan en centros de salud privados y los datos disponibles de ambos estudios no permiten aclarar el efecto de este factor ${ }^{19}$. Al analizar los resultados hay que tener presente que el grupo de estudio estuvo representado por niños que consultan a hospitales, lo que sesga negativamente las presentaciones atípicas, que se describen principalmente a propósito de búsquedas dirigidas, mediante marcadores serológicos, en grandes grupos de población, que frecuentemente presentan escasa sintomatología ${ }^{20,21}$. Considerando la calidad del grupo estudiado, son interesantes los hallazgos de $21 \%$ de casos atípicos y $11 \%$ de casos diagnosticados por tener un familiar directo celía$\mathrm{CO}$, ya que indican que los pediatras gastroenterólogos de los centros participantes han incorporado a su arsenal diagnóstico los criterios más recientes de como buscar y diagnosticar la enfermedad. Es deseable que esta práctica de búsqueda se amplíe a toda la comunidad pediátrica y también a los médicos de adultos. Las características de este estudio no permiten aclarar si la cifra encontrada debiera ser mayor.

El tiempo transcurrido hasta el diagnóstico fue inesperadamente largo, promedio 2,8 años, P 2575:1,3-7,5 años en el grupo total y mediana de 6 y 46 meses en niños bajo y sobre 10 años, respectivamente (Tabla 1); este retraso se puede deber a un bajo índice de sospecha en el equipo de salud o a dificultad para acceder a la confirmación del diagnóstico (serología y biopsia). Chile se encuentra en un estado de postransición epidemiológica ${ }^{22}$ y la desnutrición energético-proteica primaria está prácticamente ausente en Santiago, por lo cual es poco probable que la enfermedad se confunda con otras condiciones; el hecho de que se diagnosticaran presentaciones extraintestinales también sugiere que existe conciencia de la enfermedad en el equipo médico que debe buscarla. Más aún, una vez realizados los exámenes de EMA y TTG, el tiempo transcurrido para confirmar el diagnóstico fue corto, no más de una semana (datos no mostrados). Esto sugiere que el sistema de detección y diagnóstico previo a la consulta en el centro hospitalario es insuficiente en el sistema de salud público y debiera mejorarse.

Actualmente, el consenso internacional es que los EMA y TTG son las herramientas serológicas recomendadas para la evaluación diagnóstica ${ }^{5,23}$, de manera que resulta discutible que a 13\% de los pacientes se les realizara AGA en vez de EMA o TTG. En cuatro pacientes deficientes de IgA, los EMA fueron negativos, asociación que ha sido reportada como la principal causa de falla de este test ${ }^{24}$; en los restantes 4 casos de EMA negativo $(6,2 \%)$, la falla podría estar asociada a la edad del paciente, ya que se ha descrito previamente que la sensibilidad del examen en este grupo de edad es menor $^{25}$; justamente se ha argüido que a esta edad podría justificarse mantener el uso de AGA. Fue inesperado que la mayoría de los centros contaran con algún test serológico para el diagnóstico pero no para el seguimiento; esto crea una situación difícil de entender como criterio de manejo de un grupo de pacientes crónicos, que necesariamente requieren seguimiento a largo plazo.

La proporción de niños diagnosticados debido a antecedentes familiares positivos (11\%) fue más del doble de lo encontrado en un estudio previo efectuado 7 años atrás, en el que esta cifra fue de $5 \%$. Al igual que en los casos previos, todos éstos tuvieron alteraciones moderadas a graves en la mucosa intestinal, correspondiendo a formas silentes $^{26}$. Llama la atención en los estudios publicados en pacientes chilenos que no se hayan detectado casos con daños leves de la mucosa intestinal. De la sintomatología clínica, al igual que lo descrito en otros estudios, la diarrea crónica fue el síntoma más frecuente ${ }^{27}$. En estudios de individuos de riesgo o asintomáticos diagnosticados mediante rastreo, Hoffenberg ha reportado que el retraso pondoestatural sería una característica típica de pacientes que se diagnostican mediante este tipo de estudios, lo que el autor relaciona a que el diagnóstico es más tardío ${ }^{28}$; esto contrasta con los resultados de nuestro estudio, en que la falla pondoestatural fue frecuente tanto en los pacientes menores como los de mayor edad. De las presentaciones atípicas 
observadas en este estudio, cabe comentar al adolescente de 15 años que se presentó como dispepsia tipo ulcerosa y anemia asociada, que no presentaba otros síntomas intestinales, y cuya serología y hallazgos histológicos en la biopsia confirmaron la presencia de enfermedad celíaca. La revisión bibliográfica sólo reveló una publicación en que se comenta que los síntomas dispépticos son más frecuentes en población celíaca que en población general ${ }^{29}$.

En todos los centros participantes hubo la intención de realizar una segunda biopsia; sin embargo, debido a que un alto número de pacientes no seguía la dieta adecuadamente ésta se logró obtener sólo en un tercio de los pacientes. La persistencia de las lesiones intestinales fue un hallazgo frecuente en nuestros pacientes que se supone que están en dieta sin gluten, que no parece cambiar en el transcurso de los años (experiencia personal y opinión recogida entre los gastroenterólogos infantiles, Rama de Gastroenterología, Sociedad de Pediatría, no publicado). Históricamente, esto se ha atribuido al alto consumo de pan y productos derivados del trigo en nuestro medio, pero hoy en día, el grado de educación de la población ha mejorado y eso ha traído como consecuencia mayor conciencia de la enfermedad y motivación para realizar el tratamiento en forma estricta; sin embargo, los datos obtenidos de las historias clínicas sugieren que las transgresiones involuntarias continúan teniendo alta frecuencia. Aunque no diseñado para obtener información sobre la calidad de la dieta ingerida por los pacientes evaluados, el estudio reveló un dato interesante: entre las causas de las transgresiones apareció que aquellas más frecuentes eran por consumo de alimentos elaborados, cuyo con-

\section{REFERENCIAS}

1. Fassano A. Clinical Presentation of Celiac Disease in the Pediatric Population. Gastroenterology 2005; 128: s68-s73.

2. KAGNOFF MF. Overview and pathogenesis of celiac disease. NIH Consensus Development Conference on Celiac Disease 2004; 19-21.

3. Green P, Bana J. Celiac disease. The Lancet 2003; 362: 383-91. tenido de gluten era incierto. Esto pone en relieve la necesidad de mejorar el contenido del etiquetado de los alimentos, un aspecto que sin duda amerita ser evaluado en más detalle en futuros estudios.

En resumen, en los últimos 5 años, en la población infantil de Santiago atendida en los hospitales que participaron en el estudio, las presentaciones típicas predominaron en los menores de 10 años, las formas atípicas se diagnosticaron en $21 \%$ del total de casos y $11 \%$ de los casos se evaluaron por el antecedente de tener un familiar directo con enfermedad celíaca, lo cual sugiere que los nuevos criterios de detección y diagnóstico se están incorporando en nuestra comunidad de profesionales de la salud. Dos hallazgos podrían tener implicancias de salud pública: que los anticuerpos (EMA y TTG) debieran ser incorporados de rutina al proceso de diagnóstico y de seguimiento para pacientes con enfermedad celíaca en hospitales públicos, ya que su carencia lleva a un retraso importante en el diagnóstico y castiga la calidad del seguimiento; y segundo, que es importante mejorar el etiquetado de alimentos, ya que las transgresiones alimenticias involuntarias aparecen como la dificultad más frecuentemente mencionada por los pacientes para seguir adecuada y seguramente la dieta en la vida moderna.

\section{Agradecimientos}

Los autores agradecen a los colegas y otros profesionales de los centros participantes por su gentileza al poner a nuestra disposición las fichas clínicas y otros datos de los pacientes incluidos en el estudio.
4. Abdulkarim AS, Murray J. The diagnosis of celiac disease. Aliment Pharmacol Ther 2003; 17: 987-95.

5. Rostom A, Dube C, Cranney A, Saloojee N, Sy R, Garritty C ET al. The diagnostic accuracy of serologic tests for celiac disease: a systematic review. Gastroenterology 2005; 128: s38-s46.

6. Kagnoff MF. Celiac disease pathogenesis, the plot thickens. Gastroenterology 2002: 123: 939-43.

7. Fasano A, Berti I, Gerarduzzi T, Not T, Colletti RB, Drago S ET al. Prevalence of celiac disease in at- 
risk and not-at-risk groups in the United States. Arch Intern Med 2003; 163: 286-92.

8. Lo W, Sano K, Lebwohl B, Diamond B, Green PH. Changing presentation of adult celiac disease. Dig Dis Sci 2003; 48: 395-8.

9. Rostom AlaA. Incidence and prevalence of celiac disease. NIH Consensus Development Conference on Celiac Disease 2004; 57-9.

10. Castano L, Blarduni E, Ortiz L, Nunez J, Bilbao JR, Rica I et al. Prospective Population Screening for Celiac Disease. High Prevalence in the first years of life. J Pediatr Gastroenterol Nutr 2004; 39: 80-4.

11. Fassano A, Catassi C. Current Approaches to Diagnosis and Treatment of Celiac Disease: A Evolving Spectrum. Gastroenterology 2001; 120: 636-51.

12. Ferguson A, Arranz E, O'Mahony S. Clinical and pathological spectrum of coeliac disease-active, silent, latent, potential. Gut 1993; 34: 150-1.

13. Green PHR. The many faces of celiac disease: Clinical presentation of celiac disease in adult population. NIH Consensus Development Conference on Celiac Disease 2004; 65-8.

14. Green PHR, Stavropoulos SN, Panagi SG, Goldstein SL, Mcmahon DJ, Absan H et al. Characteristics of adult celiac disease in the USA: results of a national survey. Am J Gastroenterol 2001; 96: 126-31.

15. West J, Logan RF, Hill PG, Lloyd A, Lewis S, HuBbard R ET AL. Seroprevalence, correlates and characteristics of undetected celiac disease in England. Gut 2003; 52: 960-5.

16. Revised criteria for diagnosis of celiac disease. Report of Working Group of Society of Paediatric Gastroenterology and Nutrition. Arch Dis Child 1990; 65: 909-11.

17. When is a coeliac? Report of a working group of the United European Gastroenterology Week in Amsterdam, 2001. Eur J Gastroenterol Hepatol 2001; 13: 1123-8.

18. Guideline for the diagnosis and treatment of Celiac Disease in Children: Recommendations of the North American Society for Pediatric Gas- troenterology, Hepatology and Nutrition. J Pediatr Gastroenterol Nutr 2005; 40: 1-19.

19. http//www.minsal.cl/ici/nutricion/nutricion.htlm

20. Hill I, Fasano A, Schwartz R, Counts D, Glock M, Horvath K. The prevalence of celiac disease in at -risk group of children in the United States. J Pediatr 2000; 136: 86-90.

21. Hoffenberg EJ, MacKenzie T, Barriga KJ, Eisenbarth GS, Bao F, HaAs JE et al. A prospective study of the incidence of childhood celiac disease. J Pediatr 2003; 143: 308-14.

22. Albala C, Vio F. Epidemiological transition in Latin America: the case of Chile. Public Health 1995; 109: 431-42.

23. Hill ID. What are the sensitivity and specificity of serological tests for celiac disease? Do sensitivity and specificity vary in different populations? $\mathrm{NH}$ Consensus Development Conference on Celiac Disease 2004; 27-31.

24. Cataldo F, Lio D, Marino V, Picarelli A, Ventura A, Corazza GR. IgG1 antiendomysium and IgG antitissue transglutaminase (anti-tTG) antibodies in celiac patients with IgA deficiency. Gut 2000; 47: 366-9.

25. Kewiecen J, Karczewska K, Lukasik M, Kasner J, Dyduch A, ZABKa A ET AL. Negative results of antiendomysial antibodies: long term follow up. Arch Dis Child 2005; 90: 41-2.

26. Araya M, Mondragón A, Pérez Bravo F, Roessler Jl, Alarcon T, Ríos G et al. Celiac disease in Chilean population carrying Amerindian Traits. J Pediatr Gastroenterol Nutr 2000; 31: 381-6.

27. Dogancy T, Bozkurt S. Celiac disease with various presentations. Pediatrics International 2004: 46: 693-6.

28. Hoffenderg EJ, Emery lM, Barriga KJ, Bao F, Taylor J, Eisenbarth GS et al. Clinical features of children with screening-identified evidence of celiac disease. Pediatrics 2004; 113: 1254-9.

29. Bardella MT, Minoli G, Ravizza D, Radaelli F, Velio P, Quatrini M eT AL. Increased prevalence of celiac disease in patients with dyspepsia. Arch Intern Med 2000; 160: 1489-91. 